pag

Business School

WORKING PAPER SERIES

Working Paper

2014-347
Optimal Positioning in Financial Derivatives under Mixture Distributions

R. Hentati-Kaffel

J.L. Prigent

http://www.ipag.fr/fr/accueil/la-recherche/publications-WP.html

IPAG Business School

184, Boulevard Saint-Germain

75006 Paris

France 


\title{
Optimal Positioning in Financial Derivatives under Mixture Distributions*
}

\author{
R. Hentati-Kaffel ${ }^{\dagger}$ and J.L. Prigent ${ }^{\ddagger}$
}

May 2014

\begin{abstract}
In this paper, we study and extend the optimal portfolio positioning problem introduced by Brennan and Solanki (1981) and by Leland (1980). For that purpose, we introduce mixtures of probability distributions to model the log returns of financial assets. In this framework, we provide and analyze the general solution for log return with mixture distributions, in particular for the mixture Gaussian case. Our solution is characterized for arbitrary utility functions and for any risk neutral probability. Moreover, we illustrate the solution for a CRRA utility and for the minimal risk-neutral probability. Lastly, we compare our solution with the optimal portfolio within ambiguity. Our results have significant implications to improve the management of structured financial portfolios.

JEL classifications: G11; G02; C61

Keywords: Portfolio optimization; Mixture distributions; Ambiguity.
\end{abstract}

\footnotetext{
${ }^{*}$ We thank also participants of theThird International Symposium in Computational Economics and Finance (ISCEF) in Paris, April 10-12, 2014, for their useful comments and suggestions.

${ }^{\dagger}$ University of Paris 1 Panthéon-Sorbonne, CES, France. E-mail: rania.kaffel@univparis1.fr

‡IPAG Business School, 184 Bd Saint-Germain, 75006, Paris, France and ThEMA, University of Cergy-Pontoise, 33 Bd du Port, 95011, Cergy-Pontoise, France.

Tel: 331342561 72, FaX: 331342562 33, e-mail: jean-luc.prigent@u-cergy.fr
} 


\section{Introduction}

Extreme market swings of recent years have been a rude reminder that investors must be wary of unanticipated volatility spikes and large losses. They are also at odds with the assumption that returns are normally distributed. A useful rule of thumb to overcome these modeling deficiencies is to introduce mixtures of probability distributions in order to improve the estimation of asset returns and in particular to better take account of risks. Mixture models arise in many applications and in particular in finance because they provide a convenient and flexible statistical tool to better estimate multivariate distributions. Titterington et al. (1985) and Basford and McLachlan (1988) have established the main properties about the statistical inference of mixture models. These mixtures are introduced to decompose the overall density of a given random variable into a weighted linear combination of probability density functions (pdf) which belong to a given family of parametric distributions.

Typically, mixtures are introduced to take account of randomness about the right probability distribution to describe asset returns. In that case, the coefficient of the mixture corresponding to a given law is the probability that this distribution is the most convenient one. Many different methods have been applied in estimating the parameters of Mixture Distributions (MD), in particular Normal Mixture Distributions (NMD). One of these is the Expected Maximization algorithm (EM). This method is based on the maximum likelihood (see Basford and McLachlan, 1985; Leroux, 1992). This method has been used in many applications such as neural networks, clustering and signal processing. The EM algorithm (Dempster et al., 1977) is an iterative procedure to find the maximum of likelihood functions. ${ }^{1}$

The financial literature illustrates many applications of such mixtures. For example, in the case of a finite mixture of Gaussian distributions, Ritchey (1990) has introduced a NMD to involve stylized facts of non-normal returns characterized by fat-tailed and skewed distributions. In this case, we can evaluate options by using weighted sums of Black-Scholes (B-S) solutions. Melick and Thomas (1997) consider that the distribution of crude oil during the Gulf crises could be derived from a mixture of three lognormal distributions. Then, they estimate the implied pdf of prices for futures from American option prices. Other studies as in Brigo and Mercurio (2000) prove that the risk-neutral prices can be modeled by such mixture distributions. Brigo et al. (2002) assume that the local volatility corresponds to a weighted sum of deterministic volatilities and prove that this mixture describes a leptokurtic price process in a complete market. Alexander (2004) tests a binomial NMD parameterization to capture the short-term effect and extends the model to consider also the long-term smile effect. This calibration has been tested to fit Euro-US dollar exchange rates.

Bellalah and Prigent (2002) decompose the empirical returns distribution as in Bellalah and Lavielle (2002) to emphasize different phases of the market (a

\footnotetext{
${ }^{1}$ The first application of such mixtures was given by Pearson (1894).
} 
sudden fall in the bear part, a possibility of high increase in the bull phase and stable market) to evaluate standard and exotic options. Explicit formulas of the "Greeks" are then determined and compared with those obtained in the Black and Scholes models. Infinite mixture models are also associated to many ARCH type models and also to Lévy processes used to model the financial asset returns. In fact, if the jump component of the underlying asset is a Poisson process with finite intensity, then the asset return is an infinite mixture of Gaussian distributions (see Cont and Tankov, 2003; Lesne and Prigent, 2001). Mixture models are also introduced in portfolio optimization modeling and performance measurement. Hentati and Prigent (2011) examine the maximization of a very large class of performance measures within mixture probability distributions (including in particular the Kappa measures).

The aim of this paper is to contribute to the previous literature by solving the optimal positioning problem in the presence of mixture of distributions. Such problem has been addressed in the partial equilibrium framework by Brennan and Solanki (1981) and by Leland (1980). Our findings are based on the static assumption. In this sense, we consider an investor who wants to maximize the expected utility of his terminal wealth. Indeed, due to practical constraints (liquidity, transaction costs...), financial portfolios are discretely rebalanced. For example, the portfolio is rebalanced monthly. Additionally, it is important to note that many financial products are based on contracts that must be fully specified at the initial date. This is the basis of structured portfolio management (in France, it corresponds to about seven hundred funds, the so-called "fonds à formule"). The value of the portfolio corresponds to a given function of some portfolio of common assets, which is usually called the benchmark. ${ }^{2}$ As mentioned by Carr and Madan (2001), if the financial market contains all the out-of-the-money European puts and calls of all strikes, the market is complete. This assumption is justified when there is a large number of option strikes (options written on the S\&P500 for example). This problem has been already solved explicitly for Gaussian log returns by Brennan and Solanki (1981) and Leland (1980) and further extended by Bertrand et al. (2001) when taking account of insurance constraints. We extend previous results to the case corresponding to log return having mixture distributions, in particular Gaussian mixtures. Several implications arise from the resolution of this problem. First, the optimal portfolio is characterized for arbitrary utility functions and for any choice of a particular risk neutral probability if the market is incomplete. Second, the particular case of the CRRA utility and of the minimal risk-neutral probability, introduced by Föllmer and Schweizer (1991) is scrutinized. ${ }^{3}$ Third, under the risk-neutral probability, the solution is examined when the distribution of the risky asset return is a Gaussian mixture. Finally, and under the same conditions, the optimal shape of the portfolio payoff is determined in the

\footnotetext{
${ }^{2}$ Introduced by Leland and Rubinstein (1976), the portfolio insurance theory also considers portfolio payoffs which are functions of a benchmark.

${ }^{3}$ The minimal risk-neutral probability plays a key role in determining the locally quadratic risk-minimizing strategies. I is used also in various other frameworks as well.
} 
case of optimal portfolio profile with ambiguity.

The paper is organized as follows. Section 2 recalls basic properties about mixture distributions, in particular various examples of Gaussian mixtures. Basic financial examples are also provided. In Section 3, the optimal portfolio is determined in the general optimal positioning framework, using the standard expected utility maximization. When the mixture is due to the ambiguity with respect to values of the probability distribution parameters, we compare also our optimal solution to the optimal portfolio within ambiguity as introduced by Gilboa and Schmeidler (1989). This approach allows to take account of aversion to ambiguity. These results are detailed for CRRA utility functions. Section 4 concludes. Some of the proofs are gathered in Appendix.

\section{Mixture distributions}

\subsection{Empirical studies}

The Expected Maximization (EM) approach is a general iterative optimization algorithm for maximizing a likelihood score function. This method has been studied by Redner and Walker (1984), Basford and McLachlan (1985) and Leroux (1992). It is based on two steps: The E-step and the M-step. However, the determination of a global maximum is a more complex issue, since the likelihood may degenerate. Due to this drawback, Hathaway (1985) considers a constrained Maximum Likelihood (ML) method and Redner and Walker (1984) introduce the EM algorithm. The main advantage of this method allows the increases of the log-likelihood function for each iteration of the EM until a stationary point has been reached.

For financial data, Melick and Thomas (1997) prove that the assumption of a three Gaussian mixture for the oil price was justified during the Golf's war. Bellalah and Lavielle (2002) show also that a three Gaussian mixture is a good approximation of the empirical distribution for main stock financial indices. They consider two methods: the first one is purely parametric and based on the kernel estimation; the second one uses the (ML) and (EMA) methods. A specific algorithm is also introduced to identify the regime changes.

\subsection{Definitions and general properties}

Assume that each observation corresponds to a realization of a random vector $\left(X_{1}, \ldots, X_{n}\right)$, with respective cumulative distribution functions (cdf) $F_{1}, \ldots, F_{n}$. Suppose, for instance, that each random component $X_{i}$ has a distribution with parameter $\theta_{i}$ and that $\lambda_{i}$ denotes the $i$-weight of the mixture. Let $\Phi$ the global mixture parameter:

$$
\Phi=\left(\lambda_{1}, \ldots, \lambda_{n} ; \theta_{1}, \ldots, \theta_{n}\right) .
$$


Then, the probability density function (pdf) associated to this mixture distribution is given by:

$$
f(x, \Phi)=\sum_{i=1}^{n} \lambda_{i} f\left(x, \theta_{i}\right)
$$

where $f(x, \Phi)$ denotes the pdf of the mixture distribution. The weighting system $\left(\lambda_{i}\right)_{i}$ corresponds to a convex combination. We have:

$$
\sum_{i=1}^{n} \lambda_{i}=1 \text { and } \forall i \in\{1, \ldots, n\}, \lambda_{i}>0 .
$$

One interpretation of such a mixture is the following one: let $Y$ be a discrete random variable with probability distribution defined as follows:

$$
P(Y=i)=\lambda_{i}, \text { for } i=1, \ldots, n .
$$

Assume for example that the conditional distribution of the vector $X$ knowing $Y$ is Gaussian and defined by:

$$
\mathcal{L}_{X}^{Y=i}=\mathcal{N}\left(m_{i}, \Sigma_{i}\right) .
$$

Then, the pdf of $X$ satisfies: for any $x \in \mathbb{R}^{d}$,

$$
f_{X}(x)=\sum_{i=1}^{n} \lambda_{i} \frac{1}{\sqrt{(2 \pi)^{d}\left|\Sigma_{i}\right|}} \exp \left\{-\frac{1}{2}\left(x-m_{i}\right)^{T} \Sigma_{i}^{-1}\left(x-m_{i}\right)\right\} .
$$

Thus, we get a Gaussian mixture with global parameter $\Phi=\left\{\lambda_{i}, m_{i}, \Sigma_{i}\right\}_{i=1}^{n}$ since:

1. $\lambda_{i}>0, i=1, \ldots, n$ and

$$
\sum_{i=1}^{n} \lambda_{i}=1
$$

2. $m_{i} \in \mathbb{R}^{d}$ and $\Sigma_{i}$ is a definite and symmetric matrix $(d \times d)$.

Previous example is a particular case of finite mixture distributions. Other cases can be introduced (see Everit and Hand, 1981; McLachlan and Peel, 2000). Infinite mixture distributions can also be considered. First, the random variable $Y$ may have a countable set of values. Second, specific models can also be considered. For instance, to overcome some shortcomings of the Black-Scholes model, in particular the impossibility to justify the smile effect, stochastic volatility models have been introduced by Hull and White (1987) and further extended for example by Heston (1993). In that case, the log return has a mixture distribution (by conditioning with respect to the stochastic volatility). To take account of sudden high variations, jumps can be introduced in the risky asset dynamics, modelled for example by a Poisson process independent from a standard Brownian motion. Again, the log return distribution is a mixture (by conditioning with respect to both the number an amplitudes of jumps). Other infinite Gaussian mixture distributions are also associated to Arch type models in discrete-time or to switching models (see Frühwirth-Schnatter, 2006). 
An infinite mixture distribution corresponds for instance to a pdf given by:

$$
f(x, Y)=\int f(x, y) g(y) d y,
$$

where $g($.$) itself is a pdf.$

Suppose for example that the conditional distribution of the vector $X$ knowing $Y$ is given by:

$$
\mathcal{L}_{X}^{Y=y}=\mathcal{N}\left(m_{y}, \Sigma_{y}\right) .
$$

Then, we deduce that the pdf of $X$ satisfies: for any $x \in \mathbb{R}^{d}$,

$$
f_{X}(x)=\int \frac{1}{\sqrt{(2 \pi)^{d}\left|\Sigma_{y}\right|}} \exp \left\{-\frac{1}{2}\left(x-m_{y}\right)^{T} \Sigma_{y}^{-1}\left(x-m_{y}\right)\right\} g(y) d y,
$$

where $g($.$) is the pdf of Y$. We conclude that $X$ has a Gaussian mixture distribution.

The pdf of such mixture is illustrated in next figure for the S\&P 500 index (monthly returns from 30th January 1998 to 31 May 2011).

Figure 1 provides the fitting of the frequency histogram by a Gaussian pdf (Mean about $0.2 \%$ and variance about $0.23 \%$ ).

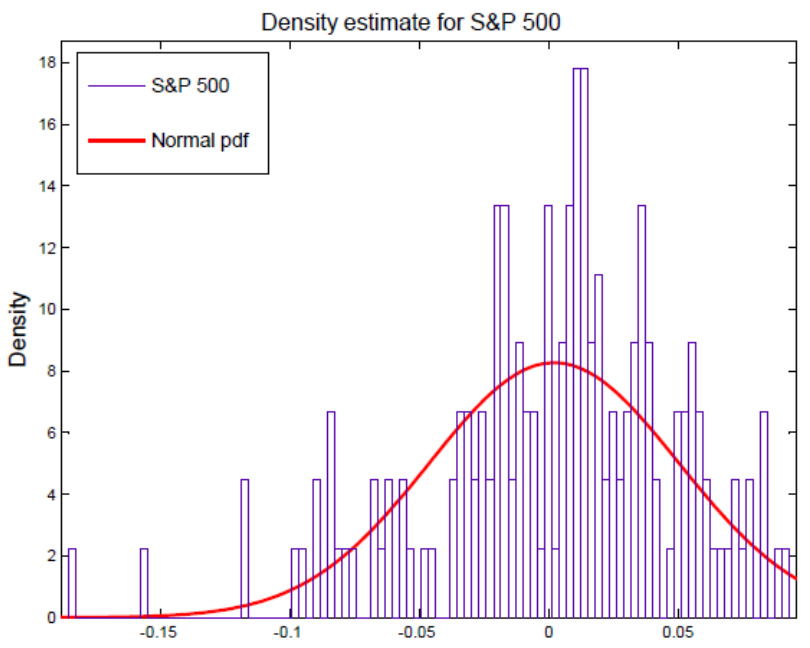

Figure 1. S\&P 500 histogram

Figure 2 illustrates the approximation by a two-Gaussian mixture and its comparison with the empirical pdf. The two Gaussian distributions $\mathcal{N}\left(m_{i}, \sigma_{i}^{2}\right)$ satisfy: $m_{1}=-3.7 \%, \sigma_{1}^{2}=0.31 \%$ and $m_{2}=1.84 \%, \sigma_{2}^{2}=0.11 \%$. The weights are given by: $\lambda_{1} \simeq 30 \%$ and $\lambda_{2} \simeq 70 \%$. 


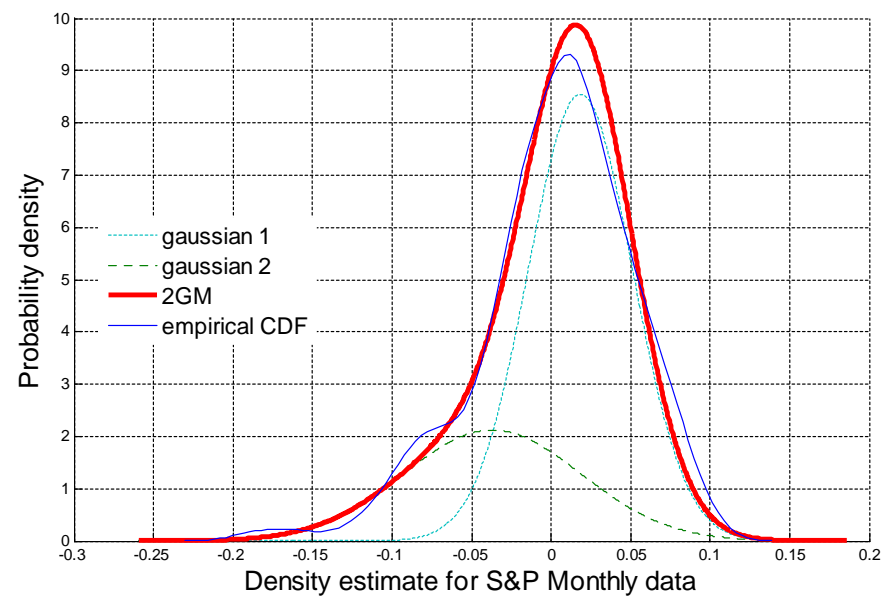

Figure 2. Two-Gaussian mixture

Finally, Figure 3 provides the three-Gaussian mixture approximation with $m_{1}=-4.96 \%, \sigma_{1}^{2}=0.26 \%, m_{2}=0.94 \%, \sigma_{2}^{2}=0.064 \%$ and $m_{3}=6.52 \%, \sigma_{3}^{2}=$ $0.023 \%$. The weights are given by: $\lambda_{1} \simeq 25.15 \%, \lambda_{2} \simeq 61.53 \%$ and $\lambda_{3} \simeq 13.32 \%$.

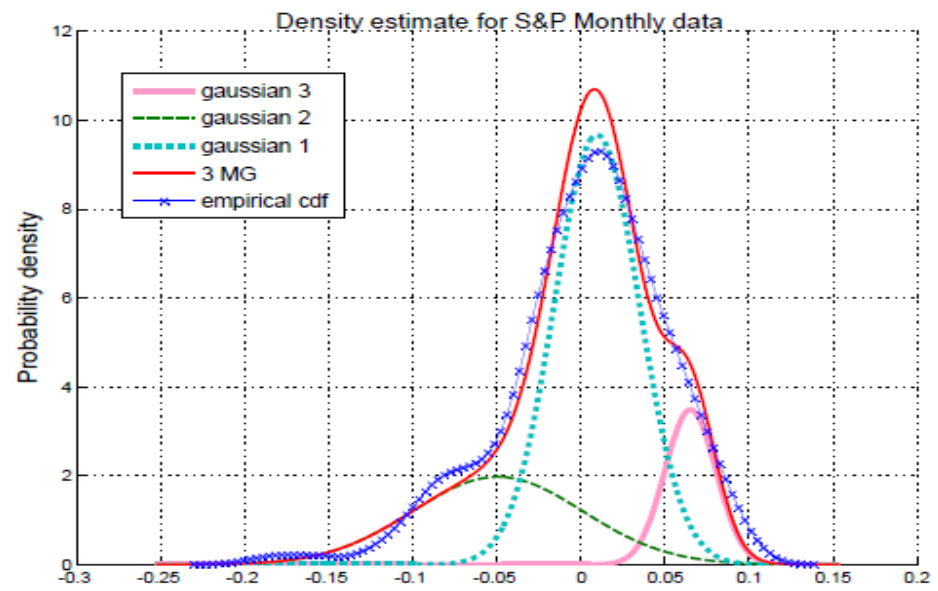

Figure 3. Three-Gaussian mixture

\subsection{Basic example}

We suppose that the underlying asset dynamic is such that:

$$
\frac{d S_{t}}{S_{t}}=\mu_{Y} d t+\sigma_{Y} d W_{t},
$$


where $Y$ denotes a random discrete variable, independent from the Brownian motion $W$. Using these assumptions, we get:

$$
S_{t}=S_{0} \exp \left[\left(\mu_{Y}-\frac{1}{2} \sigma_{Y}^{2}\right) t+\sigma_{Y} W_{t}\right] .
$$

The random variable $Y$ can be viewed as a factor that determines the drift and volatility of the risky asset. The variable $Y$ can be for instance a macroeconomic variable that is exogenous with respect to the financial market. It can also correspond to randomness of trading frequency (as proved in Appendix 1), which is in line with order book dynamics. Such situation arises from momentaneous suspension of balance of supply and demand. Another interpretation of variable $Y$ comes from ambiguity about the choice of probability distribution parameters.

We get a finite Gaussian mixture, since the logarithm $X_{t}$ of the return of $S_{t}$ satisfies:

$$
\begin{aligned}
P\left[X_{t} \leq x\right] & =P\left[m_{Y} t+\sigma_{Y} W_{t} \leq x\right] \\
& =\sum_{i=1}^{n \text { or } \infty} P\left[m_{y_{i}} t+\sigma_{y_{i}} W_{t} \leq x \mid Y=y_{i}\right] P\left[Y=y_{i}\right]
\end{aligned}
$$

where $m_{Y}=\mu_{Y}-\frac{1}{2} \sigma_{Y}^{2}$.

Thus, the pdf $f_{X_{t}}$ of $X_{t}$ is given by:

$$
f_{X_{t}}(x)=\frac{\partial P\left[X_{t} \leq x\right]}{\partial x}=\sum_{i=1}^{n \text { or } \infty} \frac{\partial P\left[m_{y_{i}} t+\sigma_{y_{i}} W_{t} \leq x\right]}{\partial x} P\left[Y=y_{i}\right] .
$$

By identifying $P\left[Y=y_{i}\right]$ to the coefficient $\lambda_{i}$ and by setting $m_{y_{i}}=m_{i}$, $\sigma_{y_{i}}=\sigma_{i}$, we deduce that the pdf $f_{X_{t}}$ of $X_{t}$ is a convex combination of Gaussian pdf $f\left(x, \theta_{i, t}\right)$ since we have:

$$
f_{X_{t}}(x)=\sum_{i=1}^{n \text { or } \infty} \lambda_{i} f\left(x, \theta_{i, t}\right) \text { with } \theta_{i, t}=\left(m_{i} t ; \sigma_{i} \sqrt{t}\right) .
$$




\section{Optimal positioning}

\subsection{General payoff function}

The optimal portfolio positioning problem has been addressed by Leland (1980), and Brennan and Solanki (1981). The portfolio value is defined as a function of a given benchmark. Its payoff maximizes the investor's expected utility. In what follows, we solve the optimal positioning problem when the risky logreturn distribution is a mixture of distributions, in particular a Gaussian mixture. The mixture is modelled by a random variable $Y$, as illustrated in previous section. The investor maximizes the expected utility of his wealth at maturity. Additionally, this latter one is a function of both the risky asset value $S_{T}$ at maturity $T$ and of the conditioning random variable $Y$ that determines the weighting of the mixture.

$$
\operatorname{Max}_{h} E\left[U\left[h\left(S_{T}, Y\right)\right]\right],
$$

where $h\left(S_{T}, Y\right)$ denotes the portfolio value at maturity $T$ and the utility function $U$ is assumed to be twice differentiable, strictly concave and non-decreasing.

\subsubsection{The risk-neutral probability.}

In what follows, we assume that the random variable $Y$ has a finite or countable set of values ${ }^{4}$. The pdf of $S_{T}$ is a mixture given by:

$$
f_{S_{T}}(s)=\sum_{i=1}^{n \text { or } \infty} \lambda_{i} f\left(s, y_{i}\right),
$$

with $P\left[Y=y_{i}\right]=\lambda_{i}$ and where $f\left(s, y_{i}\right)$ denotes the conditional pdf of $S_{T}$ evaluated at time $s$, knowing $Y=y_{i}$.

We assume that the option prices are computed by taking account of the random variable $Y$. It means that all investors agree on the additional randomness due to $Y$. In that case, the pdf $g$ of $\frac{d Q}{d P}$ with respect to the $\sigma$-algebra generated by $S_{T}$ and $Y$ has the form $g\left(S_{T}, Y\right)$ and satisfies the following relations:

$$
\begin{aligned}
g(., .) & \geq 0 \text { (positivity) } \\
\sum_{i=1}^{n \text { or } \infty} \lambda_{i} \int f\left(s, y_{i}\right) g\left(s, y_{i}\right) d s & =1(g \text { is a pdf) } \\
\sum_{i=1}^{n \text { or } \infty} \lambda_{i} \int s f\left(s, y_{i}\right) g\left(s, y_{i}\right) d s & =S_{0} e^{r T} \text { (risk neutrality). }
\end{aligned}
$$

\footnotetext{
${ }^{4}$ The case where $Y$ has a continuous distribution with pdf can be considered as well. In that case, the pdf of $S_{T}$ can be expressed as in Relation (3).
} 
A special case: The density $g$ corresponds to the density of the minimal martingale measure. The concept of minimal equivalent martingale measure $\widehat{P}$ has been introduced by Föllmer and Schweizer (1991) to obtain hedging strategies which are optimal for quadratic risk-minimization. Schweizer (1992) shows that the induced valuation process $\hat{V}_{0}=E_{\widehat{P}}[H]$ can be viewed as the intrinsic value process of the claim $H$ and that it corresponds to a certain risk-neutral approach to option valuation (see Appendix 2 for explicit determination).

\subsubsection{The optimal portfolio profile}

We have to solve the following optimization problem, under the budget constraint:

$$
\begin{aligned}
& \operatorname{Max}_{h} E\left[U\left[h\left(S_{T}, Y\right)\right]\right], \\
& \text { with } \\
V_{0}= & e^{-r T} E\left[h\left(S_{T}, Y\right) g\left(S_{T}, Y\right)\right] .
\end{aligned}
$$

Proposition 1 Under previous assumptions, the optimal portfolio value is given by:

$$
h^{*}\left(S_{T}, Y\right)=J\left[\eta g\left(S_{T}, Y\right)\right],
$$

where $J$ denotes the inverse of the marginal utility $U^{\prime}$ and $\eta$ is the Lagrange parameter associated to the budget constraint.

Proof. To simplify the presentation, we suppose as usual that the function $h$ fulfils:

$$
\sum_{i=1}^{n \text { or } \infty} \lambda_{i} \int h^{2}\left(s, y_{i}\right) f\left(s, y_{i}\right) d s<\infty .
$$

Setting $X_{T}=\left(S_{T}, Y\right)$, it means that $h \in L^{2}\left(R^{2}, P_{X_{T}}(d s, d y)\right)$ which is the set of the measurable functions with squares that are integrable on $R^{2}$ with respect to the joint distribution $P_{X_{T}}(d s, d y)$.

A new functional $\Phi_{U}$ is associated to the utility function $U$. It is defined on the space $L^{2}\left(R^{2}, P_{X_{T}}(d s, d y)\right)$ by:

For any $Z \in L^{2}\left(R^{2}, P_{X_{T}}(d s, d y)\right), \Phi_{U}(Z)=E_{P_{X_{T}}}[U(Z)]$.

$\Phi_{U}$ is usually called the Nemitski functional associated with $U$ (see for example Ekeland and Turnbull (1983) for definition and basic properties). From the properties of the utility function $U$, the Nemitski functional $\Phi_{U}$ is concave and differentiable on $L^{2}\left(R^{2}, P_{X_{T}}\right)$ (the Gâteaux-derivative exists).

Additionally, the budget constraint is a linear function of $h$. So there exists exactly one solution $h^{*} . h^{*}$ is the solution of $\frac{\partial L}{\partial h}=0$ where the Lagrangian $L$ is defined by:

$L(h, \lambda)=\int_{R^{2}}[U(h(s, y))] P_{X_{T}}(d s, d y)+\eta\left(V_{0}-\int_{R^{2}} h(s, y) g(s, y) P_{X_{T}}(d s, d y)\right)$, 
where $\eta$ is the Lagrange multiplier associated to the budget constraint. Therefore, $h^{*}$ satisfies: $U^{\prime}\left(h^{*}\right)=\eta g$ which implies $h^{*}=J(\eta g)$.

Therefore, the optimal payoff is a function of both variables $S_{T}$ and $Y$. From a practical point of view, it means that hedging such portfolio would imply to trade on options written in particular on $Y$, for example to use options on the volatility $\left(\right.$ here $\left.\sigma_{Y}\right){ }^{5}$

\subsubsection{The optimal portfolio profile with ambiguity}

Here, we assume that the investor has more ambiguity about future market evolutions. Thus he faces uncertainty about probability distribution parameters and introduce a family of distributions instead of an unique one. Contrary to mixtures modelling, he does not match a given probability to each parameter. For example, for ambiguity about normal distributions $\mathcal{N}(\mu, \sigma)$, the investor can assume that $\mu$ and $\sigma$ respectively belong to subsets $[\mu, \bar{\mu}]$ and $[\underline{\sigma}, \bar{\sigma}]$. But he does not introduce a specific probability distribution on these latter subsets.

The notion of "ambiguity" has been introduced by Ellsberg (1961). Gilboa and Schmeidler (1989) have considered the so-called "maxmin expected utility preferences", which assume the existence of multiple priors. The maxmin expected utility criterion of Gilboa and Schmeidler (1989) corresponds to the following optimization problem:

$$
\operatorname{Max}_{h \in \mathcal{H}} \operatorname{Min}_{P \in \Delta}\left(E_{P}\left[U\left(h\left(S_{T}\right)\right)\right]\right),
$$

where $\mathcal{H}$ denotes the set of possible payoffs. The set of multiple probability priors $\Delta$ correspond to ambiguity with respect to parameter $y$ of a given probability distribution $P_{y}$ which corresponds to the law when the random variable $Y$ takes the value $y$ (see basic example). However, the main difference with previous mixture case is that the weights $\lambda_{i}$ are not taken into account: the probability distribution of $Y$ has no impact on the final result except its support.

Proposition 2 If $E_{P_{y}}\left[U\left(h\left(S_{T}, Y\right)\right)\right]$ is continuous with respect to parameter $y$ of $P_{y}$ for all payoffs $h$ in a given set $\mathcal{H}$ and if $\Delta$ is a compact set, then the optimal solution $h_{A}^{*}$ of Problem (13) corresponds to an optimal solution of the standard expected utility maximization for a given parameter $y^{*}$.

Proof. See Ben Ameur and Prigent (2013).

Remark 3 Contrary to the mixture case, the optimal portfolio under ambiguity corresponds to an optimal standard portfolio for a given value of the random variable $Y$. For example, if the distribution of the risky asset return $S_{T} / S_{0}$ is Lognormal conditionally to $Y$, then the optimal portfolio under ambiguity is determined within a lognormal framework whereas the optimal portfolio within mixture corresponds to a mixture of Gaussian distributions, thus with skewness and fatter tails.

\footnotetext{
${ }^{5}$ See Carr and Madan (2001) and Prigent (2007) for the determination of the hedging strategy in the standard static case.
} 


\subsection{Basic example}

\subsubsection{Optimal positioning for the basic example}

Consider the risky asset dynamics as defined in (4):

$$
\frac{d S_{t}}{S_{t}}=\mu_{Y} d t+\sigma_{Y} d W_{t}
$$

where $Y$ denotes a random variable, independent from the Brownian motion $W$.

Denote:

$$
\begin{aligned}
& m_{Y}=\mu_{Y}-\frac{1}{2} \sigma_{Y}^{2} ; \theta_{Y}=\frac{\mu_{Y}-r}{\sigma_{Y}} \text { (Sharpe ratio), } \\
& a_{Y}=-\frac{1}{2} \theta_{Y}^{2} T+\frac{\theta_{Y}}{\sigma_{Y}} m_{Y} T ; \kappa_{Y}=\frac{\theta_{Y}}{\sigma_{Y}} ; \chi_{Y}=e^{a_{Y}}\left(S_{0}\right)^{\kappa_{Y}} .
\end{aligned}
$$

Then, we have:

$$
W_{T}=\frac{\ln \left(\frac{S_{T}}{S_{0}}\right)-m_{Y} T}{\sigma_{Y}} .
$$

We consider the minimal martingale measure $\widehat{P}$ to price options. The $\sigma$-algebra $\mathcal{F}$ is generated by the Brownian motion $W$ and the random variable $Y$. We deduce (see Appendix 2) that the pdf $g\left(S_{T}, Y\right)$ of $\frac{d \widehat{P}}{d P}$ with respect to the $\sigma$-algebra generated by $Y$ and $W_{T}$ is given by:

$$
g(s, y)=\chi_{y} s^{-\kappa_{y}} .
$$

Note that it corresponds also to special case (4). Indeed, knowing $Y=y$, we recover the standard risk-neutral density of the Black and Scholes model.

Therefore, from Relation (12), we deduce that the optimal solution is equal to:

$$
h^{*}\left(S_{T}, Y\right)=J\left[\eta \chi_{Y} S_{T}^{-\kappa_{Y}}\right],
$$

where $\eta$ is the Lagrange parameter associated to the budget constraint.

For instance, assume that the utility function is a power function $U(x)=$ $\frac{x^{1-\gamma}}{1-\gamma}$ (CRRA case) with relative risk aversion $\gamma>0$ and $\gamma \neq 1$.

Corollary 4 The optimal solution for the CRRA case is given by:

$$
h^{*}\left(S_{T}, Y\right)=c \cdot \chi_{Y}^{-\frac{1}{\gamma}} \cdot S_{T}^{\frac{\kappa_{Y}}{\gamma}},
$$

where the power $\frac{\kappa_{Y}}{\gamma}$ of $S_{T}$ is equal to the Sharpe type ratio $\kappa_{Y}=\frac{\mu_{Y}-r}{\sigma_{Y}^{2}}$ times the inverse of the relative risk aversion $\gamma$. Applying budget constraint, the coefficient $c$ is equal to

$$
c=\frac{V_{0} e^{r T}}{E\left[\left(\chi_{Y} S_{T}^{-\kappa_{y}}\right)^{\frac{\gamma-1}{\gamma}}\right]} .
$$

Note that:

$$
E\left[\left(\chi_{Y} S_{T}^{-\kappa_{y}}\right)^{\frac{\gamma-1}{\gamma}}\right]=E\left[\exp \left(\frac{1}{2} \theta_{Y}^{2} T \frac{1-\gamma}{\gamma^{2}}\right)\right]
$$




\subsubsection{Concavity-Convexity of the payoff for the CRRA case}

Depending in particular on the value of $Y$, the power $\frac{\kappa_{Y}}{\gamma}$ is higher than 1 or not. Therefore, the higher the Sharpe type ratio or the smaller the relative risk aversion, the more convex the optimal payoff as function of the risky asset. Indeed, the investor wants a convex payoff if the financial market has a good performance (high Sharpe type ratio) and/or his relative risk aversion is low. Such behavior allows to benefit from potential market rises. On the contrary, if the financial market is not sufficiently favorable (small Sharpe type ratio) and/or his relative aversion is high, then the investor prefers to get higher returns for bearish market than for bullish market. More precisely, we have:

Corollary 5 The optimal payoff is convex as soon as $\kappa_{Y}>\gamma$. Thus, the probability to get a convex payoff is equal to $P\left[\kappa_{Y}>\gamma\right]=P\left[\frac{\mu_{Y}-r}{\sigma_{Y}^{2}}>\gamma\right]$. Consider the particular case corresponding to randomness of trading frequency (see Appendix 1), where functions $\mu($.$) and \sigma($.$) are defined by: \mu(y)=\mu . y$ and $\sigma(y)=\sigma \cdot \sqrt{y}$. Then, the probability to get a convex payoff is equal to $P\left[\left(\mu-\sigma^{2} \gamma\right) Y>r\right] .^{6}$

Next figure illustrates three main cases: (numerical assumptions: volatility $\sigma=20 \%, S_{0}=100, V_{0}=1000, T=1$ year and finally relative risk aversion $\gamma=2)$.

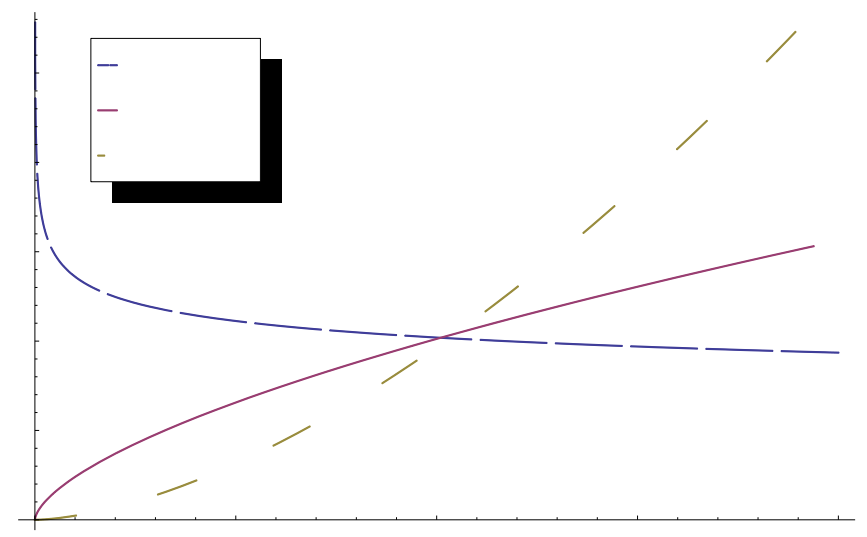

Figure 4. Optimal portfolio payoffs as function of $\mu_{Y}$.

- If $\mu$ is relatively small $(\mu=1 \%)$, the optimal positioning leads to a decreasing payoff since the investor expect small returns of the risky asset.

- If $\mu$ is moderate $(\mu=7 \%)$, the optimal positioning leads to an increasing and concave payoff since the investor expect standard return values of the risky asset.

\footnotetext{
${ }^{6}$ Assume for instance that the distribution of $Y$ is uniform on $[0.5 ; 1]$ and $\mu=18 \%, \sigma=$ $17 \%, r=3 \%$. Then the probability to get a convex payoff is equal to 1 for an aggressive investor $(\gamma=2)$, to 0.3 for a moderate investor $(\gamma=5)$ and finally to 0 for a conservative investor $(\gamma=10)$.
} 
- If $\mu$ is relatively high $(\mu=10 \%$ ), the optimal positioning leads to an increasing and convex payoff since the investor expect high returns of the risky asset and wants to benefit from potential market rises.

\subsubsection{The optimal portfolio profile with ambiguity for the CRRA case}

In what follows, we illustrate the role of the ambiguity for a CRRA utility $U(v)=\frac{v^{1-\gamma}}{1-\gamma}$ and Gaussian mixture as described in (4). The set of multiple priors $\Delta$ correspond to ambiguity with respect to parameter $y$. We assume that $\Delta$ corresponds to $y \in[y, \bar{y}]$ and that both functions $\mu(y)$ and $\sigma(y)$ are continuous with respect to $y$ on $[\bar{y}, \bar{y}]$.

Consider the maximin expected utility criterion of Gilboa and Schmeidler (1989).

Corollary 6 The optimal payoff $h_{A}^{*}$ with respect to the maximin expected utility criterion is a power function of the risky asset. It is defined by:

$$
h_{A}^{*}(s)=d \cdot \chi_{y^{*}}^{-\frac{1}{\gamma}} \cdot S_{T}^{\frac{\kappa_{y^{*}}}{\gamma}}
$$

where $y^{*}$ minimizes the absolute value of the Sharpe ratio $\theta(y)=\frac{\mu(y)-r}{\sigma(y)}$ and

$$
d=\frac{V_{0} e^{r T}}{E\left[\exp \left(\frac{1}{2} \theta_{y^{*}}^{2} T \frac{1-\gamma}{\gamma^{2}}\right)\right]}
$$

Proof. Applying Proposition (2), we deduce that the optimal solution $h_{A}^{*}$ is the solution of the standard expected utility criterion for one particular value $y^{*}$ in $[\underline{y}, \bar{y}]$. Thus, we have to solve the following minimization problem:

$$
\operatorname{Min}_{y \in[\underline{y}, \bar{y}]} E\left[U\left(c \cdot \chi_{y}^{-\frac{1}{\gamma}} \cdot S_{T}^{\frac{\kappa_{y}}{\gamma}}\right)\right] .
$$

Using in particular (17) and (18), this problem is equivalent to:

$$
\operatorname{Min}_{y \in[\underline{y}, \bar{y}]}\left(\frac{\left[V_{0} e^{r T}\right]^{1-\gamma}}{1-\gamma}\right) \exp \left(\frac{1}{2} \theta_{y}^{2} T \frac{1-\gamma}{\gamma}\right) \text {. }
$$

Since previous function is increasing with respect to $\theta_{y}^{2}$, this problem is equivalent to the minimization of the absolute value of the Sharpe ratio $\theta(y)$.

Note that this result is similar to result of Chen and Epstein (2002) in the continuous-time case, when $\Delta$ corresponds to the set $-M \leq \theta_{y} \leq M$, with $M$ non negative constant. The main difference is that here we consider that the risk-neutral probability takes also account of the ambiguity. For the particular case corresponding to randomness of trading frequency (see Appendix 1), we have: $\mu(y)=\mu . y$ and $\sigma(y)=\sigma \cdot \sqrt{y}$. Thus, we have to minimize $\left|\theta_{y}\right|=\left|\frac{\mu \cdot y-r}{\sigma \cdot \sqrt{y}}\right|$. Assuming for instance that $\mu . \underline{y}>r$, the optimal solution corresponds to $y^{*}=\underline{y}$. 
In what follows, we illustrate numerically the optimal payoffs for the three cases: Gaussian, ambiguity and mixture. We use previous S\&P 500 data. Recall that, for the Gaussian case, the mean return is about $0.2 \%$ and the variance is about $0.23 \%$ ). For the three-Gaussian mixture case, we get: $m_{1}=-4.96 \%, \sigma_{1}^{2}=$ $0.26 \%, m_{2}=0.94 \%, \sigma_{2}^{2}=0.064 \%$ and $m_{3}=6.52 \%, \sigma_{3}^{2}=0.023 \%$. The weights are given by: $\lambda_{1} \simeq 25.15 \%, \lambda_{2} \simeq 61.53 \%$ and $\lambda_{3} \simeq 13.32 \%$. The risk-free rate $r$ is assumed to be equal to $0.02 / 12$ (monthly return). We consider two relative risk aversion levels: weak risk aversion $(\gamma=0.3)$; moderate risk aversion $(\gamma=5)$. We assume that the initial risky asset value $S_{0}$ is equal to 100 and that the initial portfolio value $V_{0}$ is equal to 1000 .

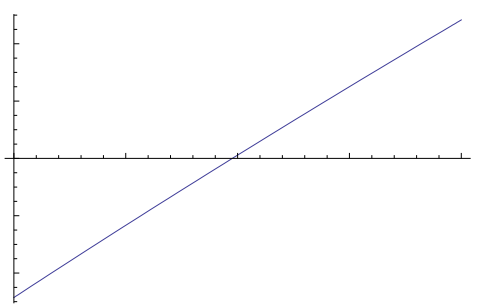

Gaussian case, $\gamma=0.3$

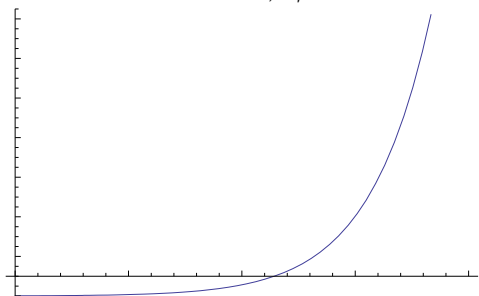

Ambiguity case, $\gamma=0.3$

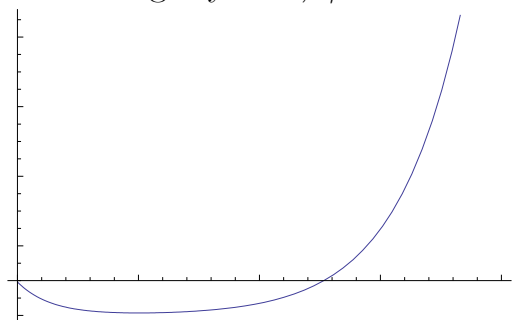

Mixture case, $\gamma=0.3$

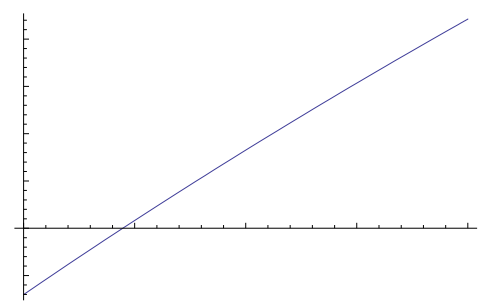

Gaussian case, $\gamma=5$

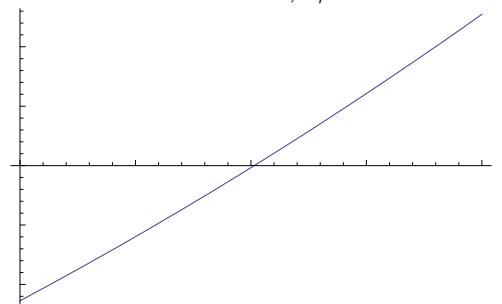

Ambiguity case, $\gamma=5$

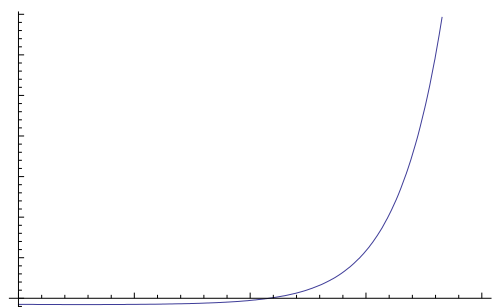

Mixture case, $\gamma=5$

Figure 5. Comparison of the optimal payoffs.

For the Gaussian case, the optimal payoff is almost linear on interval [90;110], since $\kappa=0.507$, which implies that exponents $\kappa / 0.3 \simeq 1.7$ and $\kappa / 5 \simeq 0.1$. When the investor is weakly risk averse, he seeks to relatively high returns when the risky asset rises, while accepting losses when the risky asset drops. If his risk aversion is moderate, he prefers to get smaller returns for bullish market, which allows him to get better returns for bearish market.

For the ambiguity case, the optimal payoff corresponds to the Gaussian distribution $\mathcal{N}\left(m_{2}=0.94 \%, \sigma_{2}^{2}=0.064 \%\right)$, since it has the smallest absolute 
value $\left|\theta_{y}\right|$. For weakly risk aversion, the investor searches for higher returns when the risky asset rises than for moderate risk aversion. Consequently, he can loose much more when the market is bearish.

For the mixture case, we provide the expectation of the three optimal payoffs corresponding to the three Gaussian distributions of the mixture, weighted by their respective coefficients $\lambda_{i}$. For weak aversion, we note that the optimal payoff is decreasing then increasing. This is mainly due to the following property: for the first Gaussian distribution $\mathcal{N}\left(m_{1}=-4.96 \%, \sigma_{1}^{2}=0.26 \%\right)$, weighted by $\lambda_{1} \simeq 25.15 \%$, the optimal payoff is decreasing (since $m_{1}<r$ ), while, for the others, it is increasing. Thus, the expectation of the optimal payoffs is alternatively decreasing and increasing.

\section{Conclusion}

In this paper, we have adopted a mixture of probability distributions to deal with the optimal portfolio positioning problem. First, we have tested the suitability of these mixtures to fit non linear financial assets. An empirical example based on the monthly returns of the S\&P 500 index proves that a three-Gaussian mixture approximation leads to an improvement in the precision of the density estimate. Second, we have analyzed the optimal portfolio positioning introduced by Brennan and Solanki (1981) and by Leland (1980) for Gaussian log returns. Our analysis extends these researches as follows: i) we solve the general portfolio positioning problem and examine in particular the CRRA case when the risk-neutral probability corresponds to the minimal measure; ii) we illustrate the solution for a CRRA utility and for the minimal risk-neutral probability; iii) we introduce the notion of ambiguity and we compare the expectation of the optimal portfolio profile in the case of the mixture distribution with the standard no mixture case and with the optimal payoff when the investor has an aversion to ambiguity. As illustrated, the introduction of mixtures distributions can generate optimal payoffs that are not always an increasing function of the underlying risky asset. Thus, our results show that mixture of distributions can have significant implications on the portfolio management. It allows improving management of financial structured products by better taking account of market risk and by providing a better adequacy to investor's risk attitude. 


\section{References}

[1] Alexander, C. (2004). Normal mixture diffusion with uncertain volatility: modelling short and long term smile effects. Journal of Banking and Finance, 28, 2957-2980.

[2] Ansel J.P., Stricker C. (1992). Lois de martingale, densités et décomposition de Föllmer-Schweizer. Annales de l'Institut Henri Poincaré, Vol. 28, 3, 375392.

[3] Bacmann, J.-F. and Scholz, S., (2003). Alternative performance measures for hedge funds. AIMA Journal, June.

[4] Basford, K.E., and McLachlan, G.J. (1985). Likelihood estimation with normal mixture models. Applied Statistics, 34, 282-289.

[5] Bellalah, M. and Lavielle, M. (2002). A simple decomposition of empirical distributions and its applications in asset pricing. Multinational Finance Journal, 6, 99-130.

[6] Bellalah, M. and Prigent, J.-L. (2002). Pricing standard and exotic options in the presence of a finite mixture of Gaussian distributions. International Journal of Finance, 13, 1974-2000.

[7] Ben Ameur, H. and J.-L. Prigent. (2013). Optimal portfolio positioning under ambiguity. Economic Modelling, 34, 89-97.

[8] Bertrand, P., Lesne, J.P. and Prigent, J.-L. (2001). Gestion de portefeuille avec garantie : l'allocation optimale en actifs dérivés. Finance, 22, 7-35.

[9] Brennan, M.J. and Solanki, R., (1981). Optimal portfolio insurance. Journal of Financial and Quantitative Analysis, 16, 279-300.

[10] Brigo, D., and Mercurio, F., (2000). A mixed-up smile. Risk, 13, 123-126.

[11] Brigo, D., and Mercurio, F., (2002). Lognormal-mixture dynamics and calibration to market volatility smiles. International Journal of Theoretical and Applied Finance 5, 427-446.

[12] Carr, P. and Madan, D., (2001). Optimal positioning in derivative securities. Quantitative Finance, 1, 19-37.

[13] Choulli T. and Stricker, C. (1996). Unicité et existence de la loi minimale. Lecture Notes in Mathematics. Sem.Prob. XXX, 12-23.

[14] Cont, R., and Tankov, P. (2003). Financial Modelling with Jump Processes, CRC Press.

[15] Cox J., S. Ross and Rubinstein, M. (1979). Options pricing: a simplified approach. Journal of Financial Economics, 7, 229-263. 
[16] Dempster, A., Laird, N., and Rubin, D. (1977). Maximum-likelihood from incomplete data via the EM algorithm. Journal of the Royal Statistics Society., B 39, 1-38.

[17] Ekeland, I. and Turnbull, T. (1983). Infinite-Dimensional Optimization and Convexity. Chicago Lectures in Mathematics, the University of Chicago Press.

[18] Ellsberg, D. (1961). Risk, ambiguity, and the Savage axioms. Quarterly Journal of Economics, 75, 643-669.

[19] Everit, B.S., and Hand, D.J. (1981). Finite Mixture Distributions. Monographs in Applied Probability and Statistics. Chapman and Hall, London.

[20] Föllmer H., Schweizer, M. (1991). Hedging of contingent claims under incomplete information. Applied Stochastic Analysis, Stochastics Monographs,eds. M.H.A.Davis and R.J.Elliott, Gordon and Breach, Vol 5, London/New York, 389-414.

[21] Frühwirth-Schnatter, S. (2006). Finite Mixture and Markov Switching Models. Springer, New York.

[22] Gilboa, I., and Schmeidler, D. (1989). Maxmin expected utility with a nonunique prior. Journal of Mathematical Economics, 18(2), 141-153.

[23] Hathaway, R.A., (1985). Constrained formulation of maximum-likelihood estimation for normal mixture distributions. Annals of Statistics, 13, 795800.

[24] Hentati, R and Prigent, J.-L. (2011). On the maximization of financial performance measures within mixture models. Statistics and Decisions, 28, 63-80.

[25] Heston, S. L. (1993). A closed-form solution for options with stochastic volatility with applications to bond and currency options. Review of Financial Studies 6, 327-343.

[26] Hull, J. C., and White, A. (1987). The pricing of options on assets with stochastic volatilities. Journal of Finance, 42, 281-300.

[27] Leland, H. (1980). Who should buy portfolio insurance? Journal of Finance, $35,581-594$.

[28] Leroux, B.G. (1992). Consistant estimation of mixing distribution. Annals of Statistics, 20: 1350-1360.

[29] Lesne, J.-P. and Prigent, J.-L. (2001). A general subordinated stochastic process for the derivatives pricing. International Journal of Theoretical and Applied Finance, 4, 121-146. 
[30] McLachlan, G.J. and Peel, D. (2000). Finite Mixture Models. Wiley Series in Probability and Statistics. Wiley.

[31] Melick, W., and Thomas, C.P. (1997). Recovering an asset's implied pdf from option prices: An application to crude oil during the Gulf Crisis. Journal of Financial and Quantitative Analysis, 32, 91-115.

[32] Pearson, K. (1894). Contributions to the mathematical theory of evolution. Philosophical Transactions of the Royal Society. London A 185, 71-110.

[33] Prigent, J.-L. (1999). Incomplete markets: convergence of options values under the minimal martingale measure. Advances in Applied Probability, 31, 1058-1077.

[34] Prigent, J.-L. (2007). Portfolio Optimization and Performance Analysis. Chapman \& Hall, Florida.

[35] Rachev S.T., Ruschendorf, L. (1995). Models for options prices. Theory of Probability and its Applications, 39, 120-152.

[36] Redner, R.A. and Walker, H.F. (1984). Mixture densities, maximum likelihood and the EM algorithm. SIAM Review, 26, 195-223.

[37] Ritchey, R.J. (1990). Call option valuation for discrete normal mixtures. Journal of Financial Research, 13, 285-296.

[38] Schweizer, M. (1992). Mean-variance hedging for general claims. Annals of Applied Probability, 2, 171-179.

[39] Titterington, D.M., Smith, A.F.M., and Makov, U.E. (1985). Statistical Analysis of Finite Mixture Distributions. John Wiley: Chichester. 


\section{Appendix}

\section{Appendix 1. Binomial approximations with a random number of price changes.}

In what follows, we consider an extension of the well-known approximation of the Black and Scholes model by the Cox-Ross-Rubinstein (1979) model. It is based on approximations by binomial models with a random number of price changes. This framework allows to take account of the random number of stock prices changes. This feature is typically observed in financial data. The randomization of the number of price changes is based on an extension of the model of Rachev-Ruschendorf (1995), as illustrated in Prigent (1999). In discrete-time, the stock price is given by:

$$
S_{n, t}=S_{0} \prod_{k=1}^{k=N_{n}(t)}\left(1+Y_{n, k}\right),
$$

where:

1) The rates of returns $Y_{n, k}$ are equal to $\frac{\mu}{n}+T_{n, k}$ with $\mu$ constant and $\left(T_{n, k}\right)_{k}$ is a sequence of independent and identically distributed (i.i.d.) Bernoulli random variables such that:

$$
\left.P\left[T_{n, k}=\frac{u}{\sqrt{n}}\right]=p \in\right] 0,1\left[, P\left[T_{n, k}=\frac{d}{\sqrt{n}}\right]=1-p=q .\right.
$$

Parameters $u$ and $d$ are assumed to satisfy $p u+q d=0$. Denote $\sigma=\sqrt{p u^{2}+q d^{2}}$. 2) The sequence $N_{n}(t)$ of the number of changes of prices until $t$ is defined as follows. Introduce the sequence $\left(N_{n}\right)_{n}$ of integer valued random variables independent of $Y_{n, k}$ with $E\left[N_{n}\right]=n$ and $\frac{N_{n}}{n} \stackrel{\mathcal{L}}{\rightarrow} Y$ where $Y$ is a positive random variable. Consider the same subdivision $\left(T_{i}\right)_{i}$ of the interval $[0,1]$ as in RachevRuschendorf (1995): For all $i, T_{i+1}-T_{i}=\frac{1}{N_{n}}$. The stock price changes at each time $T_{i}$. Define the sequence $N_{n}(t)$ of the number of changes of prices until $t$. That is : $N_{n}(t)=\left[t N_{n}\right] . N_{n}$ is known at the first time of change of price which corresponds to the first time when $N_{n}(t)$ is different from 0 . If this first time is "immediately" after $0,{ }^{7}$ then we have $\mathbb{E}\left[N_{n}(t) \mid \mathcal{F}_{n, s}\right]=N_{n}(t)$. Theorem 4.3 in Rachev-Ruschendorf (1995) implies the convergence of the sequence $\left(S_{n, t}\right)_{n}$ to the price process $\left(S_{t}\right)_{t}$ where $S_{t}$ verifies:

$$
S_{t}=S_{0} \exp \left[\left(\mu-\frac{1}{2} \sigma^{2}\right) t Y+\sigma \sqrt{Y} d W_{t}\right]
$$

and, for $t>0, \mathcal{F}_{t}$ is equal to the $\sigma$-algebra of $\left(Y, W_{s}, s \leq t\right)$.

\footnotetext{
${ }^{7} \mathbb{E}\left[N_{n}(t) \mid \mathcal{F}_{n, s}\right]=\mathbb{E}\left[N_{n}(t) \mid N_{n}(s)\right]=N_{n}(t) I_{\left\{N_{n}(s) \neq 0\right\}}+\mathbb{E}\left[N_{n}(t)\right] I_{\left\{N_{n}(s)=0\right\}}$.

Besides, $\mathbb{P}\left[N_{n}(s)=0\right]=\mathbb{P}\left[\frac{1}{N_{n}}>s\right]$ and one has $\mathbb{P}\left[\frac{1}{N_{n}}>s\right] \rightarrow 0$ since $\frac{N_{n}}{n} \stackrel{\mathcal{L}}{\rightarrow} Y:>0$ a.s. So without loss of generality, one can assume that the first time of change of price is "immediatetly" after 0 .
} 


\section{Appendix 2. The minimal martingale measure.}

In the special case of discrete models, under a few conditions (like nondegeneracy ) on the mean-variance tradeoff process, Schweizer (1992) proves that the expectation of contingent claim $H$ with respect to the minimal measure (denoted by $E_{\widehat{P}}[H]$ ) is the value of the variance-optimal hedging strategy. It is the initial amount required for implementing a risk-minimizing strategy with terminal payoff equal to the contingent claim $H$. Usually, this strategy is not self-financing and may have a non-vanishing hedging cost. However, as soon as the minimal martingale is a true probability, this value is an actual no-arbitrage price.

All processes are defined on filtered probability spaces $\left(\Omega, \mathcal{F},\left(\mathcal{F}_{t}\right), P\right)$ indexed on $[0, T]$ and satisfying the usual conditions. When $X$ is a semimartingale and $K$ a predictable process integrable with respect to $X$, one denotes by $K . X$ the stochastic integral of $K$ with respect to $X$. Let $\mathcal{S}_{l o c}^{2}$ be the space of semimartingales $S$ such that $S_{t}^{*}=: \sup _{0 \leq s \leq t}\left|S_{s}\right|$ is locally square integrable. In what follows, the discounted underlying asset price will be denoted by $\left(S_{t}\right)_{t}$. It is a $R$-valued processs which is a special semimartingale with the canonical decomposition

$$
S=S_{0}+M+A,
$$

where $M$ is a local martingale and $A$ is a predictable process with finite variation. Let $\langle M, M\rangle$ denote the predictable quadratic variation of the martingale $M$.

Concerning the properties of existence and uniqueness of the minimal martingale measure, one uses the results of Ansel and Stricker (1992) and Choulli and Stricker (1996). The stock prices $S$ has the canonical decomposition:

$$
S=M+\lambda \cdot\langle M, M\rangle,
$$

where $\alpha$ is a predictable process that satisfies:

$$
\alpha^{2} \cdot\langle M, M\rangle<\infty
$$

Ansel and Stricker (1992) prove the following existence result: If $1-\alpha \Delta M>$ 0 a.s. then the Doleans-Dade exponential $\hat{Z}=\mathcal{E}(-\alpha . M)$ is a local positive martingale. The minimal martingale measure is a probability and its density process is given by $\mathcal{E}(-\alpha . M)$. If $P[1-\alpha \Delta M \leq 0]>0$, the minimal martingale measure is no longer a probability. However, in this last case, if $S$ is discrete, this measure is usually a signed martingale measure $\widehat{P}$ as defined in Schweizer (1992). It means that:

$\widehat{P}[\Omega]=1, \widehat{P}<<P$ on $\mathcal{F}$ with $\frac{d \widehat{P}}{d P} \in L^{2}(P)$ and $S$ is a $(\widehat{P}, \mathcal{F})$ - martingale in the sense that : $E\left[\frac{d \widehat{P}}{d P} \Delta S_{k} \mid \mathcal{F}_{k-1}\right]=0, P-$ a.s. for $k=1, \ldots, T$.

This happens for instance for the basic example. In the one-dimensional case, it is always possible to get an explicit form of the minimal martingale measure in discrete time. The basis has the form $\left(\Omega_{n}, \mathcal{F}_{n}=\left(\mathcal{F}_{n, k}\right)_{k}, P_{n}\right)$ and the canonical 
decomposition of the discounted stock price process $S_{n}=S_{0}+M_{n}+A_{n}$ is given by:

$$
\left\{\begin{array}{l}
A_{n}=\sum_{1 \leq k \leq n} E\left[S_{n, k}-S_{n, k-1} \mid \mathcal{F}_{n, k-1}\right] \\
M_{n}=\sum_{1 \leq k \leq n}\left(S_{n, k}-S_{n, k-1}-E\left[S_{n, k}-S_{n, k-1} \mid \mathcal{F}_{n, k-1}\right]\right)
\end{array}\right.
$$

The minimal martingale measure is defined by:

$$
\frac{d \widehat{P}_{n}}{d P_{n}}=\prod_{k=1}^{k=n}\left(1-\alpha_{n, k} \Delta M_{n, k}\right),
$$

where $\alpha_{n, k}=\frac{E\left[S_{n, k}-S_{n, k-1} \mid \mathcal{F}_{n, k-1}\right]}{E\left[\left(M_{n, k}-M_{n, k-1}\right)^{2} \mid \mathcal{F}_{n, k-1}\right]}$. Let $Y_{n, k}$ denote the rate of return of $S_{n, k}\left(S_{n, k}=S_{n, k-1}\left(1+Y_{n, k}\right)\right)$ and $\tilde{Y}_{n, k}$ denote the term $E\left[Y_{n, k} \mid \mathcal{F}_{n, k-1}\right]$. We get:

$$
\alpha_{n, k}=\frac{\left(\tilde{Y}_{n, k}\right)}{S_{n, k-1} \times\left(E\left[Y_{n, k}^{2} \mid \mathcal{F}_{n, k-1}\right]-\tilde{Y}_{n, k}^{2}\right)},
$$

and

$$
\Delta M_{n, k}=S_{n, k-1}\left(Y_{n, k}-\tilde{Y}_{n, k}\right)
$$

Then

$$
\alpha_{n, k} \Delta M_{n, k}=\frac{\left(\tilde{Y}_{n, k}\right)\left(Y_{n, k}-\tilde{Y}_{n, k}\right)}{\left(E\left[Y_{n, k}^{2} \mid \mathcal{F}_{n, k-1}\right]-\tilde{Y}_{n, k}^{2}\right)} .
$$

For the basic example (approximations by binomial models with a random number of price changes), we can first explicitly calculate the price under the minimal martingale measure then prove its robustness under weak convergence (see Prigent, 1999). Define the filtration $\mathcal{F}_{n, t}$ equal to the sub $\sigma$-algebra of $\left(N_{n}(t),\left(Y_{n, k}\right)_{k \leq N_{n}(t)}\right)$. This implies the following relations:

(a)

$$
S_{n, t}=S_{0} \mathcal{E}\left(R_{n, t}\right) \text { with } R_{n, t}=\sum_{k=1}^{k=N_{n}(t)} Y_{n, k} .
$$

(b) The semimartingale $R_{n, t}$ has the canonical decomposition

$$
R_{n, t}=A_{n, t}^{R}+M_{n, t}^{R},
$$

with $A_{n, t}^{R}=N_{n}(t) E\left[Y_{n, 1}\right]$ and $M_{n, t}^{R}=R_{n, t}-N_{n}(t) E\left[Y_{n, 1}\right]$.

(c) Therefore the stock price $S_{n, t}$ is equal to $A_{n, t}^{S}+M_{n, t}^{S}$ where

$$
d A_{n, t}^{S}=S_{n, t-} E\left[Y_{n, 1}\right] d N_{n}(t) \text { and } d M_{n, t}^{S}=S_{n, t-} d M_{n, t}^{R} .
$$

(d) The process $\alpha_{n, t}$ is defined by

$$
\alpha_{n, t}=\frac{1}{S_{n, \frac{[n t]-1}{N_{n}}}} \frac{E\left[Y_{n, 1}\right]}{\operatorname{Var}\left[Y_{n, 1}\right]} .
$$


The previous assumptions imply the convergence of options prices under the minimal martingale measure:

$$
\left(M_{n},\left\langle M_{n}, M_{n}\right\rangle, \alpha_{n}\right) \stackrel{\mathcal{L}\left(\mathbb{D}^{3}\right)}{\longrightarrow}(M,\langle M, M\rangle, \alpha),
$$

with

$$
M_{t}=\sigma \sqrt{Y} \int_{0}^{t} S_{u-} d W_{u},\langle M, M\rangle_{t}=\sigma^{2} Y \int_{0}^{t} S_{u}^{2} d u, \alpha_{t}=\frac{1}{S_{t-}} \frac{\mu}{\sigma^{2}} .
$$

Thus, we get the continuous-time model with its specific properties. 